\title{
Numerical Solutions of Micropolar Nanofluid over an Inclined Surface Using Keller Box Analysis
}

\author{
Khuram Rafique, ${ }^{1}$ Hammad Alotaibi, ${ }^{2}$ Taher A. Nofal, ${ }^{3}$ Muhammad Imran Anwar, ${ }^{4}$ \\ Masnita Misiran, ${ }^{5}$ and Ilyas Khan ${ }^{6}{ }^{6}$ \\ ${ }^{1}$ Department of Mathematics, University of Sialkot, Sialkot 51310, Pakistan \\ ${ }^{2}$ Department of Mathematics, College of Science, Taif University, P.O. Box 11099, Taif 21944, Saudi Arabia \\ ${ }^{3}$ Department of Mathematics and Statistics, Faculty of Science, Taif University, P.O. Box 11099, Taif 21944, Saudi Arabia \\ ${ }^{4}$ Department of Mathematics, Faculty of Science, University of Sargodha, Sargodha, Pakistan \\ ${ }^{5}$ School of Quantitative Sciences, Universiti Utara Malaysia, Sintok 06010, Kedah, Malaysia \\ ${ }^{6}$ Faculty of Mathematics and Statistics, Ton Duc Thang University, Ho Chi Minh City, Vietnam
}

Correspondence should be addressed to Ilyas Khan; ilyaskhan@tdtu.edu.vn

Received 7 October 2020; Revised 10 November 2020; Accepted 30 November 2020; Published 14 December 2020

Academic Editor: Hijaz Ahmad

Copyright (c) 2020 Khuram Rafique et al. This is an open access article distributed under the Creative Commons Attribution License, which permits unrestricted use, distribution, and reproduction in any medium, provided the original work is properly cited.

\begin{abstract}
The Brownian motion and thermophoretic impacts attained a noticeable intention of the recent researchers because these factors trigger the thermal conductivity of the nanofluid. In this study, we focus on radiation and Soret effects on a slanted stretchable sheet. Buongiorno's model is taken into account with Brownian motion and thermophoretic effects. Compatible transformations are implemented to attain the nonlinear differential equation from the boundary value PDE's. The physical quantities of practical interest are treated by graphically as well as numerically. For numerical results, the Keller box technique is applied. The numerical outcomes through tabulated magnitudes performed a good settlement with already existing results. Energy transfer rate against involved factor exhibited via graphs. Energy and mass transport rates enhance against increment in Soret factor while skin friction diminishes. Moreover, Nusselt number and Sherwood number decrease on improving inclination while skin friction increases.
\end{abstract}

\section{Introduction}

Due to the ideal potential of heat and mass exchange impacts, the nanofluids have pulled in consideration of analysts worldwide. These fluids are the mixture of nano-sized particles along with base fluids. The main purpose to mix the nanoparticles into base fluids is to enhance the thermal conductivity. Brownian motion and thermophoretic effects are two principal ideas for an abnormal upgrade of thermal conductivity. This model is becoming more widely used since it empowers us to efficiently explore different applications in the marvels of science and technology. Recently, the flow of the Casson nanofluid over an inclined surface was discussed numerically by Rafique et al. [1]. Moreover, the flow of Casson nanofluid on a slanted rotating disk was probed by Saeed et al.
[2]. In addition, Rani and Govindarajan [3] investigated the nanofluid flow through an inclined plate numerically. Nowadays, many investigators discussed the flow of nanofluid by considering different geometries [4-10].

Thermal radiations are an active part of the research which is very valuable in the latest technology, cancer cure, missiles, and nanotechnology. Pal and Roy [11] discussed the numerical impact of thermal radiation on the flow of nanofluid along the sheet. More recently, Ghadikolaei et al. [12] scrutinized Casson nanofluid flow along permeable slanted surface via a famous numerical technique. Saidulu et al. [13] considered an exponentially slanted sheet for examining the radiation influences on nanofluid flow. For further details about the literature on the flow of nanofluid by considering different geometries, see [14-24]. 
The behavior of the flow of non-Newtonian fluid is a study of keen interest of scholars and practical significance. There is a little regular and mechanical utilization of such fluids, for occasion volcanic magma, molten polymers, penetrating mud, oils, certain paints, liquid suspensions, food products, and cosmetic and numerous others. In the literature, there exist numerous numerical models with various constitutive conditions including a distinctive set of exact parameters. The micropolar liquid model is suitable for exotic oils, animal blood, fluid crystals with rigid atoms, certain organic liquids, and colloidal or suspensions solutions. The micromotion of liquid constituents, spin inertia, and the influences of the couple stresses are very noteworthy in micropolar liquids. Eringen [25] offered the philosophy of micropolar fluids based on constitution equations. Uddin [26] studied the variable electrical conductivity on the flow of micropolar liquid. Recently, Shamshuddin and Thumma discussed the flow of micropolar fluid on an inclined sheet numerically [27]. Nandhini and Ramya [28] studied the flow of micropolar fluid by incorporating the radiation effects. For some recent investigations on micropolar fluids with different impacts, see [29-34].

This work focuses on establishing the basis for a novel study on thermal investigation in solar magnetohydrodynamic nanotechnology. The approach empowers us with great flexibility to dissect the Brownian motion and thermophoretic impact on the flow of micropolar nanofluid with thermal radiations and Soret impacts on the inclined geometry. The physical quantities of practical interest such as energy exchange, velocity, skin friction, and concentration species are elaborated in graphical and tabulated form. It is worth stating there is no investigation assumed for the study under concern and all the results are new and contrast to the current outcomes with already available literature [35]. In this article, we employed the Keller box technique for numerical results. Moreover, the current results can be obtained via another numerical technique, but the Keller box method is more easier to program, friendly, and flexible. For complete detail about Keller box scheme, see [36].

\section{Problem Formulation}

Our main aim in this analysis is to examine the radiations and Soret impacts on MHD flow of micropolar nanofluid. A uniform external magnetic field of strength $B_{0}$ is considered perpendicular to the inclined surface in this study, while induced magnetic field is neglected [37]. The slanted sheet here is stretched with a stretching rate " $a$ " due to which the flow is generated. Moreover, $\omega$ is the angle taken with the vertical direction of the stretching sheet. Brownian motion and thermophoretic impacts are considered. In addition, suction or injection impacts on heat and species exchange rates are discussed via graphs.

The Lorentz force in momentum equation is expressed by $J \times B$ where

$J$ represents the current density,

$B$ denotes the total magnetic field:

$$
B=B_{0}+b \text {, }
$$

$B_{0}$ denotes the applied magnetic field,

$b$ is the induced magnetic field,

$J=\sigma(E+V \times B)$ (Ohm's law),

$\sigma$ is the electrical conductivity,

$E=0$ is the polarization charge effect,

$J=\sigma(V \times B)$,

$J \times B=\sigma(V \times B) \times B($ cross product with $B)$,

$J \times B=\sigma(V \cdot B) B-(B \cdot B) V$ (vector identity),

Using Afify [38] $\times B=\sigma B_{0}^{2} u$.

The governing equations for the study under concern are

$$
\begin{aligned}
\frac{\partial u}{\partial x}+\frac{\partial v}{\partial y}= & 0 \\
u \frac{\partial u}{\partial x}+v \frac{\partial u}{\partial y}= & \left.\frac{\mu+K_{1}^{*}}{\rho}\right) \frac{\partial^{2} u}{\partial y^{2}}+\left(\frac{K_{1}^{*}}{\rho}\right) \frac{\partial N^{*}}{\partial y} \\
& +g\left[\beta_{t}\left(T-T_{\infty}\right)-\beta_{c}\left(C-C_{\infty}\right)\right] \cos \omega \\
& -\left(\frac{\sigma B_{0}^{2}}{\rho}\right) u,
\end{aligned}
$$

$$
\begin{aligned}
u \frac{\partial N^{*}}{\partial x}+v \frac{\partial N^{*}}{\partial y}= & \left(\frac{\gamma^{*}}{j^{*} \rho}\right) \frac{\partial^{2} N^{*}}{\partial y^{2}} \\
& -\left(\frac{K_{1}^{*}}{j^{*} \rho}\right)\left(2 N^{*}+\left(\frac{\partial u}{\partial y}\right)\right),
\end{aligned}
$$

$$
\begin{aligned}
u \frac{\partial T}{\partial x}+v \frac{\partial T}{\partial y}= & \alpha \frac{\partial^{2} T}{\partial y^{2}}-\frac{1}{(\rho c)_{f}} \frac{\partial q_{r}}{\partial y} \\
& +\tau\left[D_{B} \frac{\partial C}{\partial y} \frac{\partial T}{\partial y}+\frac{D_{T}}{T_{\infty}}\left(\frac{\partial T}{\partial y}\right)^{2}\right]
\end{aligned}
$$

$$
u \frac{\partial C}{\partial x}+v \frac{\partial C}{\partial y}=D_{B} \frac{\partial^{2} C}{\partial y^{2}}+\frac{D_{T} K_{T}}{T_{\infty}} \frac{\partial^{2} T}{\partial y^{2}} .
$$

The Rosseland approximation is described by

$$
q_{r}=-\frac{4 \sigma^{*}}{3 k^{*}} \frac{\partial T^{4}}{\partial y}
$$

By employing Taylor expansion, approximate value of $T^{4}$ is given as

$$
T^{4} \cong\left(4 T_{\infty}^{3} T-3 T_{\infty}^{4}\right)
$$

By employing (6) and (7), equation (4) becomes

$$
\begin{aligned}
u \frac{\partial T}{\partial x}+v \frac{\partial T}{\partial y}= & \left(\alpha+\frac{16 \sigma^{*} T_{\infty}^{3}}{3 k^{*}(\rho c)_{f}}\right) \frac{\partial^{2} T}{\partial y^{2}} \\
& +\tau\left[D_{B} \frac{\partial C}{\partial y} \frac{\partial T}{\partial y}+\frac{D_{T}}{T_{\infty}}\left(\frac{\partial T}{\partial y}\right)^{2}\right]
\end{aligned}
$$

The subjected boundary conditions are 


$$
\begin{aligned}
u & =u_{w}(x)=a x \\
v & =V_{w}, \\
T & =T_{w}, \\
N^{*} & =-m_{0} \frac{\partial u}{\partial y}, \\
C & =C_{w}, \quad \text { at } y=0, \\
u & \longrightarrow u_{\infty}=0, \\
v & \longrightarrow 0, \\
T & \longrightarrow T_{\infty}, \quad \\
N^{*} & \longrightarrow 0, \\
C & \longrightarrow C_{\infty}, \quad \text { at } y \longrightarrow \infty .
\end{aligned}
$$

The stream function $\psi=\psi(x, y)$ for the concerned study are in the following form:

$$
\begin{aligned}
& u=\frac{\partial \psi}{\partial y} \\
& v=-\frac{\partial \psi}{\partial x}
\end{aligned}
$$

The similarity transformations are defined as

$$
\begin{aligned}
u & =\operatorname{axf}(\eta), \\
v & =-\sqrt{a v} f(\eta), \\
\eta & =y \sqrt{\frac{a}{v},} \\
N^{*} & =a x^{m} \sqrt{\frac{a(m+1) x^{m-1}}{2 v}} h(x), \\
\theta(\eta) & =\frac{T-T_{\infty}}{T_{w}-T_{\infty}}, \\
\phi(\eta) & =\frac{C-C_{\infty}}{C_{w}-C_{\infty}} .
\end{aligned}
$$

The converted form of equations (2)-(5) given by employing equation (8) is

$$
\begin{aligned}
(1+K) f^{\prime \prime \prime}+f f^{\prime \prime}-f^{\prime 2}+K h^{\prime}+\left(G r_{x} \theta+G c_{x} \phi\right) \cos \omega-M f^{\prime} & =0, \\
\left(1+\frac{K}{2}\right) h^{\prime \prime}+f h^{\prime}-f^{\prime} h-K\left(2 h+f^{\prime \prime}\right) & =0, \\
\operatorname{Pr}_{N} \theta^{\prime \prime}+f \theta \prime+N b \phi^{\prime} \theta^{\prime}+N t \theta^{\prime 2} & =0, \\
\phi^{\prime \prime}+\operatorname{Lef} \phi^{\prime}+\operatorname{SrLe} \theta^{\prime \prime} & =0,
\end{aligned}
$$

where

$$
\begin{aligned}
M & =\frac{\sigma B_{0}^{2}}{a \rho}=\frac{\nu}{D_{B}}, \\
\operatorname{Pr} & =\frac{\nu}{\alpha}, \\
N_{b} & =\frac{\tau D_{B}\left(C_{w}-C_{\infty}\right)}{\nu}, \\
N_{t} & =\frac{\tau D_{t}\left(T_{w}-T_{\infty}\right)}{\nu T_{\infty}}, \\
G r_{x} & =\frac{g \beta_{t}\left(T_{w}-T_{\infty}\right) x^{-1}}{a^{2}}, \\
R e_{x} & =\frac{u_{w} x}{v}, \\
G c_{x} & =\frac{g \beta_{c}\left(C_{w}-C_{\infty}\right) x^{-1}}{a^{2}}, \\
\operatorname{Pr} & =\frac{1}{\operatorname{Pr}\left(1+\left(\frac{4}{3}\right) N\right),} \\
S r & =\frac{D_{T} K_{T}\left(T_{w}-T_{\infty}\right)}{v T_{\infty}\left(C_{w}-C_{\infty}\right)} .
\end{aligned}
$$

where in order to make local Grashof number and local modified Grashof number free from $x$, the coefficient of thermal expansion $\beta_{t}$ and coefficient of concentration expansion $\beta_{c}$ are proportional to $x^{1}$. Hence, we assume that (see references [39-41])

$$
\begin{aligned}
& \beta_{t}=n x^{1}, \\
& \beta_{c}=n_{1} x^{1} .
\end{aligned}
$$

where $n$ and $n_{1}$ are the constants; thus $G r_{x}$ and $G c_{x}$ become

$$
\begin{aligned}
& G r=\frac{g n\left(T_{w}-T_{\infty}\right)}{a^{2}}, \\
& G c=\frac{g n_{1}\left(C_{w}-C_{\infty}\right)}{a^{2}} .
\end{aligned}
$$

The corresponding boundary conditions are

$$
\begin{aligned}
f(\eta) & =S, \\
f^{\prime}(\eta) & =1, \\
h(\eta) & =0, \\
\theta(\eta) & =1, \\
\phi(\eta) & =1, \quad \text { at } \eta=0, \\
f^{\prime}(\eta) & \longrightarrow 0, \\
h(\eta) & \longrightarrow 0, \\
\theta(\eta) & \longrightarrow 0, \\
\phi(\eta) & \longrightarrow 0, \quad \text { as } \eta \longrightarrow \infty .
\end{aligned}
$$


TAble 1: Contrast of $-\theta^{\prime}(0)$ and $-\phi^{\prime}(0)$ against $\omega=90^{\circ}, M, K, G c, N, S, S r$, and $G r=0$, with $\operatorname{Pr}=L e=10$.

\begin{tabular}{|c|c|c|c|c|c|}
\hline \multirow{2}{*}{$\mathrm{Nb}$} & \multirow{2}{*}{$N t$} & \multicolumn{2}{|c|}{ Khan and Pop [35] } & \multicolumn{2}{|c|}{ Present results } \\
\hline & & $-\theta^{\prime}(0)$ & $-\phi^{\prime}(0)$ & $-\theta^{\prime}(0)$ & $-\phi^{\prime}(0)$ \\
\hline 0.1 & 0.1 & 0.9524 & 2.1294 & 0.9524 & 2.1294 \\
\hline 0.2 & 0.2 & 0.3654 & 2.5152 & 0.3654 & 2.5152 \\
\hline 0.3 & 0.3 & 0.1355 & 2.6088 & 0.1355 & 2.6088 \\
\hline 0.4 & 0.4 & 0.0495 & 2.6038 & 0.0495 & 2.6038 \\
\hline 0.5 & 0.5 & 0.0179 & 2.5731 & 0.0179 & 2.5731 \\
\hline
\end{tabular}

TABLE 2: Values of $-\theta^{\prime}(0),-\phi^{\prime}(0)$, and $C_{f x}(0)$.

\begin{tabular}{ccccccccccccccc}
\hline$N b$ & $N t$ & $P r$ & $L e$ & $M$ & $K$ & $G r$ & $G c$ & $S r$ & $N$ & $S$ & $\omega$ & $-\theta^{\prime}(0)$ & $-\phi^{\prime}(0)$ & $C_{f x}(0)$ \\
\hline 0.1 & 0.1 & 6.5 & 5.0 & 0.1 & 1.0 & 0.1 & 1.1 & 1.0 & 1.0 & 0.5 & $45^{\circ}$ & 0.4102 & 0.8426 & 0.6211 \\
$\mathbf{0 . 5}$ & 0.1 & 6.5 & 5.0 & 0.1 & 1.0 & 0.1 & 1.1 & 1.0 & 1.0 & 0.5 & $45^{\circ}$ & 0.2823 & 1.1278 & 0.6508 \\
0.1 & $\mathbf{0 . 5}$ & 6.5 & 5.0 & 0.1 & 1.0 & 0.1 & 1.1 & 1.0 & 1.0 & 0.5 & $45^{\circ}$ & 0.2421 & 1.0143 & 0.6805 \\
0.1 & 0.1 & $\mathbf{1 0 . 0}$ & 5.0 & 0.1 & 1.0 & 0.1 & 1.1 & 1.0 & 1.0 & 0.5 & $45^{\circ}$ & 0.3728 & 1.2044 & 0.6129 \\
0.1 & 0.1 & 6.5 & $\mathbf{1 0 . 0}$ & 0.1 & 1.0 & 0.1 & 1.1 & 1.0 & 1.0 & 0.5 & $45^{\circ}$ & 0.4060 & 1.2567 & 0.6412 \\
0.1 & 0.1 & 6.5 & 5.0 & $\mathbf{1 . 0}$ & 1.0 & 0.1 & 1.1 & 1.0 & 1.0 & 0.5 & $45^{\circ}$ & 0.3668 & 0.7444 & 1.1470 \\
0.1 & 0.1 & 6.5 & 5.0 & 0.1 & $\mathbf{3 . 0}$ & 0.1 & 1.1 & 1.0 & 1.0 & 0.5 & $45^{\circ}$ & 0.4202 & 0.8715 & 0.9926 \\
0.1 & 0.1 & 6.5 & 5.0 & 0.1 & 1.0 & $\mathbf{1 . 0}$ & 1.1 & 1.0 & 1.0 & 0.5 & $45^{\circ}$ & 0.4285 & 0.8711 & 0.3154 \\
0.1 & 0.1 & 6.5 & 5.0 & 0.1 & 1.0 & 0.1 & $\mathbf{2 . 0}$ & 1.0 & 1.0 & 0.5 & $45^{\circ}$ & 0.4393 & 0.9002 & 0.2286 \\
0.1 & 0.1 & 6.5 & 5.0 & 0.1 & 1.0 & 0.1 & 1.1 & $\mathbf{2 . 0}$ & 1.0 & 0.5 & $45^{\circ}$ & 0.4783 & 1.1029 & 0.4321 \\
0.1 & 0.1 & 6.5 & 5.0 & 0.1 & 1.0 & 0.1 & 1.1 & 1.0 & $\mathbf{3 . 0}$ & 0.5 & $45^{\circ}$ & 0.3929 & 0.5100 & 0.6444 \\
0.1 & 0.1 & 6.5 & 5.0 & 0.1 & 1.0 & 0.1 & 1.1 & 1.0 & 1.0 & $\mathbf{0 . 7}$ & $45^{\circ}$ & 0.2461 & 0.8347 & 0.5748 \\
0.1 & 0.1 & 6.5 & 5.0 & 0.1 & 1.0 & 0.1 & 1.1 & 1.0 & 1.0 & 0.5 & $\mathbf{6 0}^{\circ}$ & 0.3971 & 0.8152 & 0.7807 \\
\hline
\end{tabular}

The related expressions for the skin friction coefficient $C_{f x}(0)=f^{\prime \prime}(0)$, the reduced Sherwood number $-\phi^{\prime}(0)$, and the reduced Nusselt number $-\theta^{\prime}(0)$ are demarcated as

$$
\begin{aligned}
-\theta^{\prime}(0) & =\frac{N u_{x}}{(1+(4 / 3) N) \sqrt{R e_{x}}}, \\
-\phi^{\prime}(0) & =\frac{S h_{x}}{\sqrt{R e_{x}}} \\
C_{f x} & =C_{f} \sqrt{R e_{x}} .
\end{aligned}
$$

\section{Results and Discussion}

The objective behind this section is to elaborate on the numerical results along with graphically results via tables and graphs. In order to check the validity of our numerical values with already published results (Table 1), we managed the deficiency of $G r, G c, M, N, S r, K$, and $S$, taking factor $\operatorname{Pr}=L e=10$, with $\omega=90^{\circ}$. The variations in $-\theta \prime(0)$, $-\phi^{\prime}(0) \infty$, and $C_{f x}(0)$ for physical factors $N b, N t, M, N, G r$, $G c, \omega, \operatorname{Pr}, L e, S r, S$, and $K$ are presented in Table 2. Table 2 demonstrates that the energy flux slows down by improving the Brownian motion effect. Moreover, the mass flux increases with the effect of the Brownian factor for large values. Moreover, the exchange rate of heat reduces with improving Brownian motion factor, and mass exchanges enlarge on enhancing the thermophoretic impacts. Physically, the enhancement in the Brownian motion causes the boundary layer to get thicker. Besides, skin friction shows a direct relation with Brownian motion thermophoretic effects. The magnetic effect factor boosts the skin friction against improving magnetic field magnitude. Consequently, the fluid applies a drag force on the solid boundary layer. In addition, the Nusselt number along with Sherwood number and skin friction reduces for increasing the suction parameter. The Soret effect improves the mass exchange and reduces the skin friction on enhancing the Soret impact on the mass flow.

Figures 1-8 are exhibited to demonstrate the performance of incorporated constraints that impact on the velocity sketch. Figure 1 indicates that the impact of the magnetic field creates resistance in the path of fluid flow because of the Lorentz force which reduces the velocity profile. Moreover, the angular velocity presents opposite behavior against the magnetic field factor in Figure 2. Figure 3 shows that the velocity contour upsurges against material factor $K$. Physically, increment in factor $K$ declines the viscosity and upturns the velocity. On the contrary, against a higher magnitude of the factor $K$, the angular velocity upturns (Figure 4). This demonstration corresponds with the outcomes of Rafique et al. [42]. Figure 5 represents as we enhance, the buoyancy force the velocity increases. Besides, the velocity profile shows the direct relation with $G c$ in Figure 6. In addition, the velocity field and inclination parameter correspond to an inverse relation drawn in Figure 7. Physically, by considering $\omega=0$, the gravitational force reaches its maximum value. On the contrary, in the case of $\omega=90^{\circ}$, the sheet will be in horizontal position, and that is why the power of the bouncy forces declines which is the reason behind the reduction in the velocity profile. Moreover, in Figure 8, the velocity profile enhances against the higher values of $S$. 


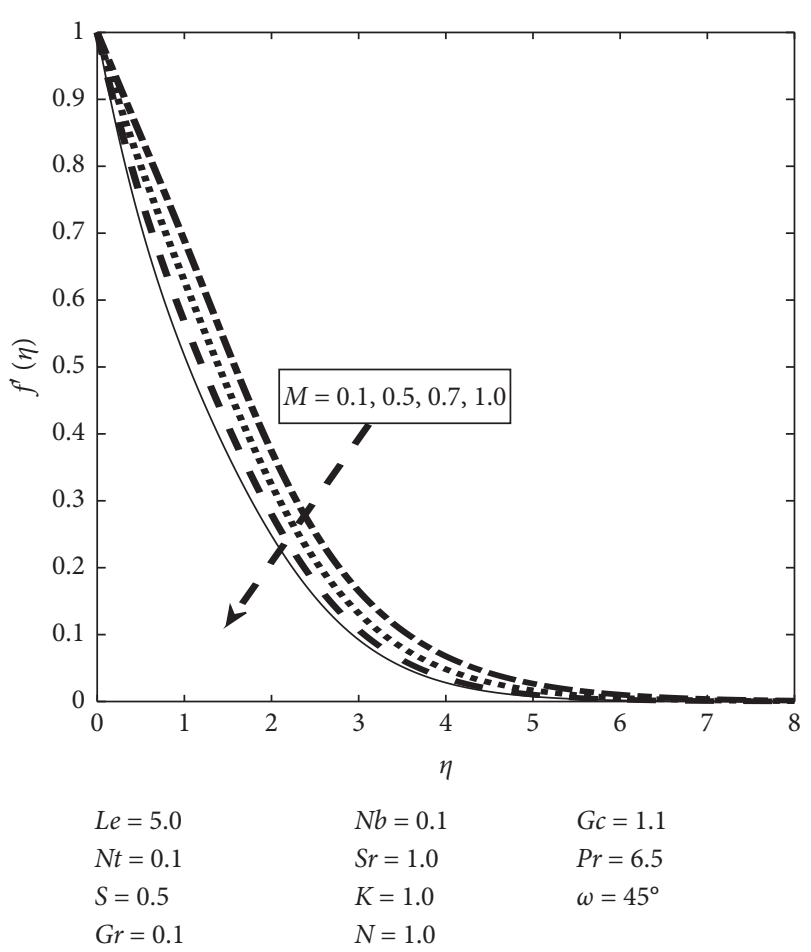

FIgURE 1: Velocity profile for several values of $M$.

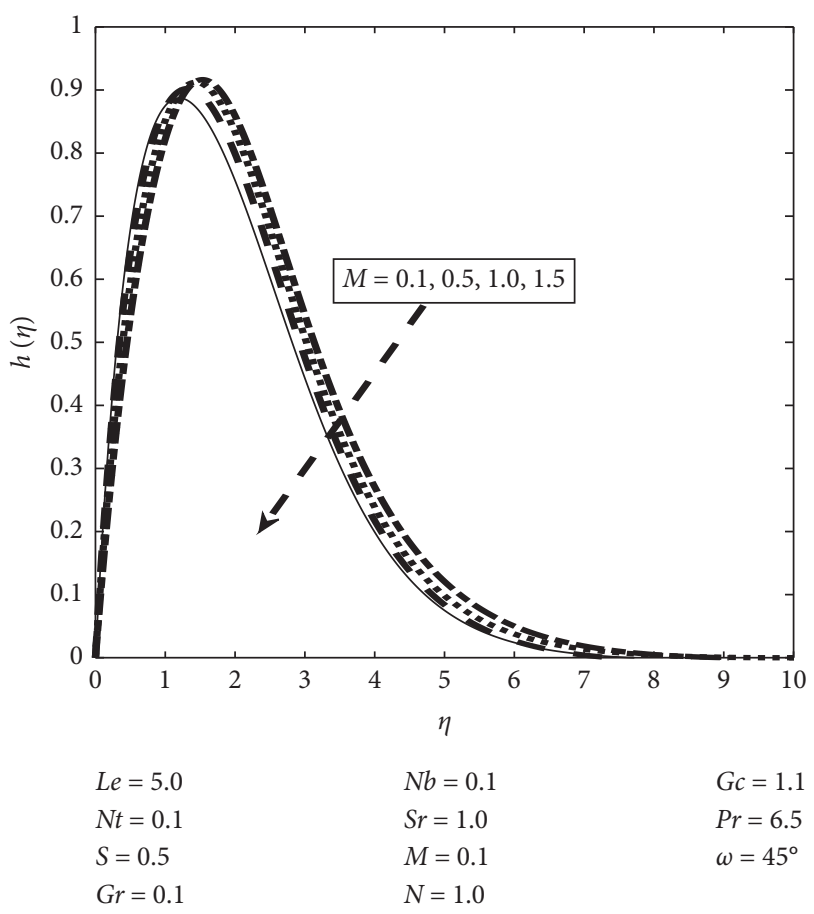

FIgURE 2: Angular velocity for several values of $M$.

3.1. Temperature. The impacts of incorporated factors in our current study against temperature profile are demonstrated in Figures 9-12. The temperature profile boosts against a stronger magnetic field because the boundary layer thickness upsurge corresponds to a higher magnetic effect (Figure 9). The Prandtl number triggers the temperature profile shown in Figure 10.

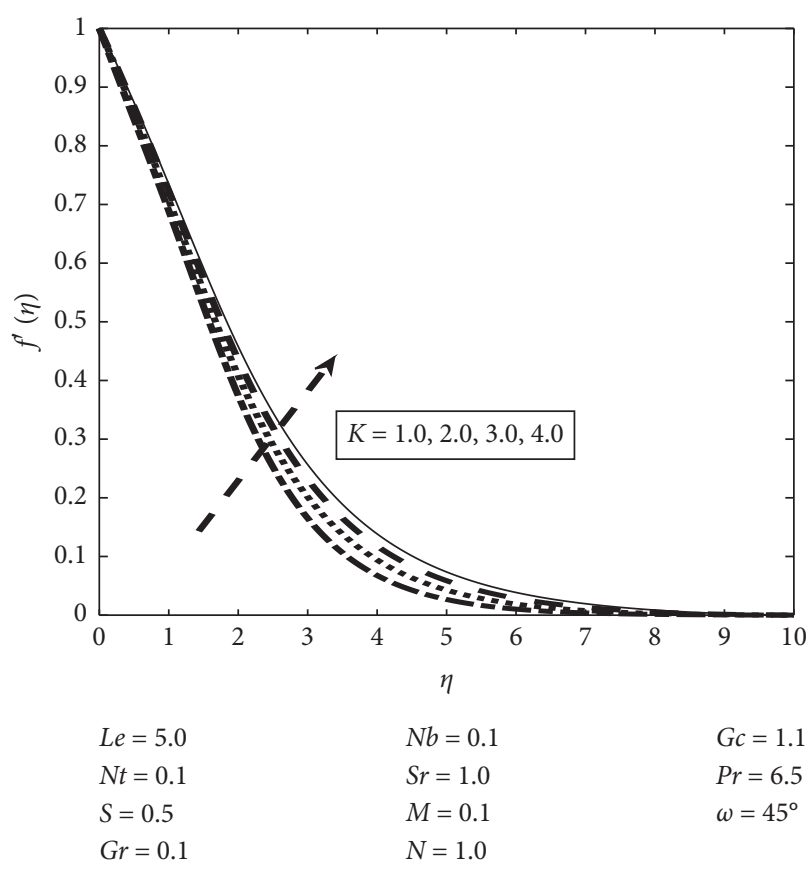

Figure 3: Velocity profile for several values of $K$.

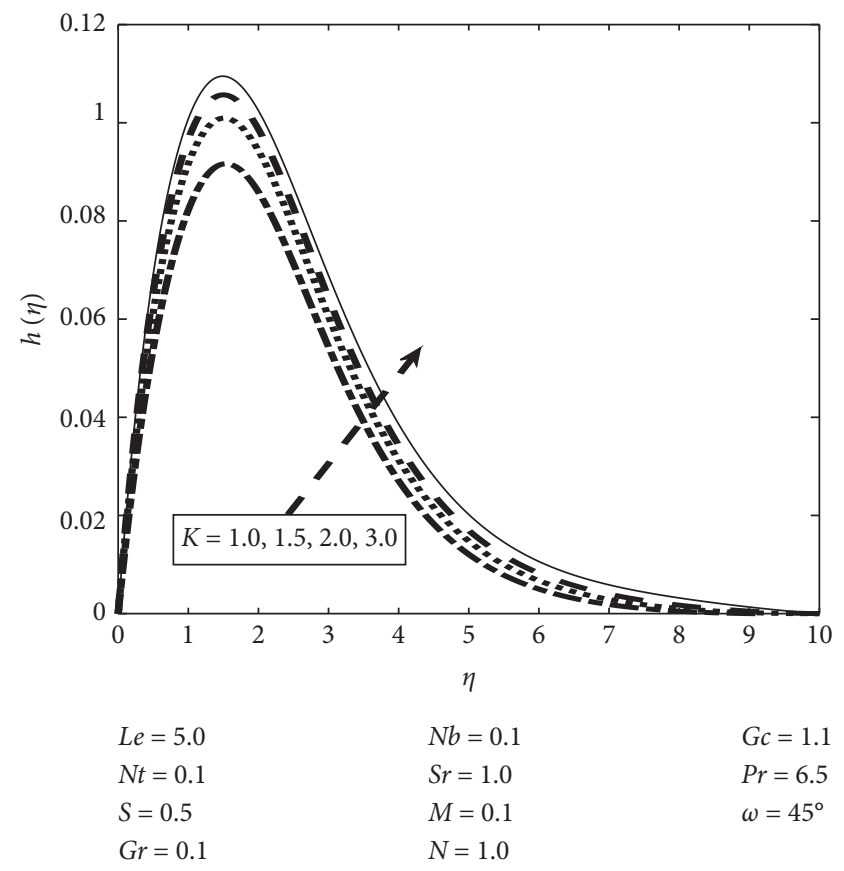

FIgURe 4: Angular velocity profile for several values of $K$.

The thermal boundary layer becomes thinner, and the thermal diffusivity becomes weaker on improving the Prandtl number steadily. The large values of the radiation effect accelerate the temperature profile as shown in Figure 11. Physically, the conductive heat exchange is greater than the radiative heat exchange, which causes reduction in boundary layer thickness and buoyancy force. The recovered outcome is the affirmative proof of the relation $q_{r}=-\left(4 \sigma^{*} / 3 k^{*}\right)\left(\partial T^{4} / \partial y\right)$. The temperature profile relates directly proportional to the Brownian 


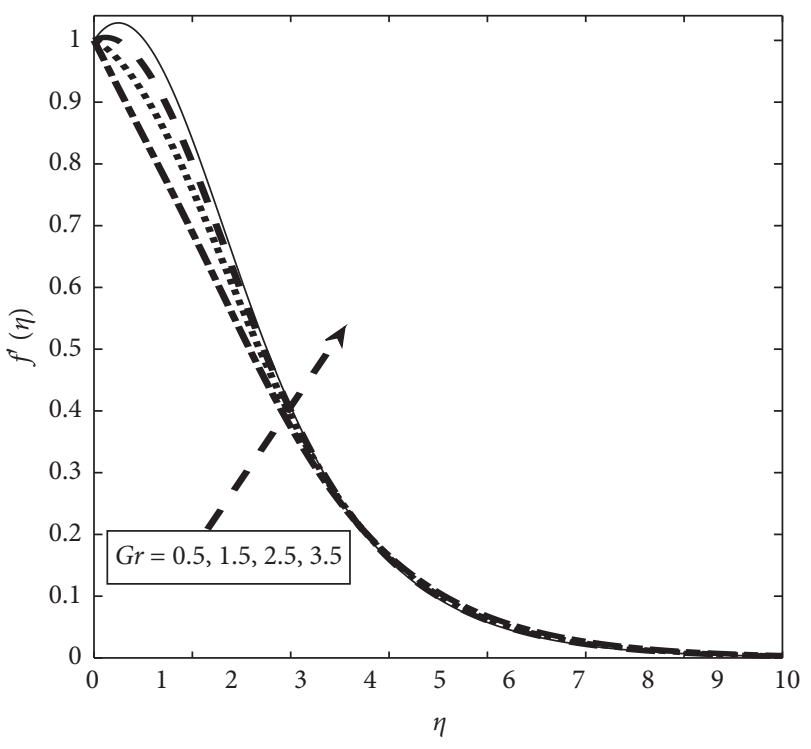

$\begin{array}{lll}L e=5.0 & N b=0.1 & G c=1.1 \\ N t=0.1 & S r=1.0 & P r=6.5 \\ S=0.5 & M=0.1 & \omega=45^{\circ} \\ K=1.0 & N=1.0 & \end{array}$

FIgURE 5: Velocity profile for several values of $G r$.

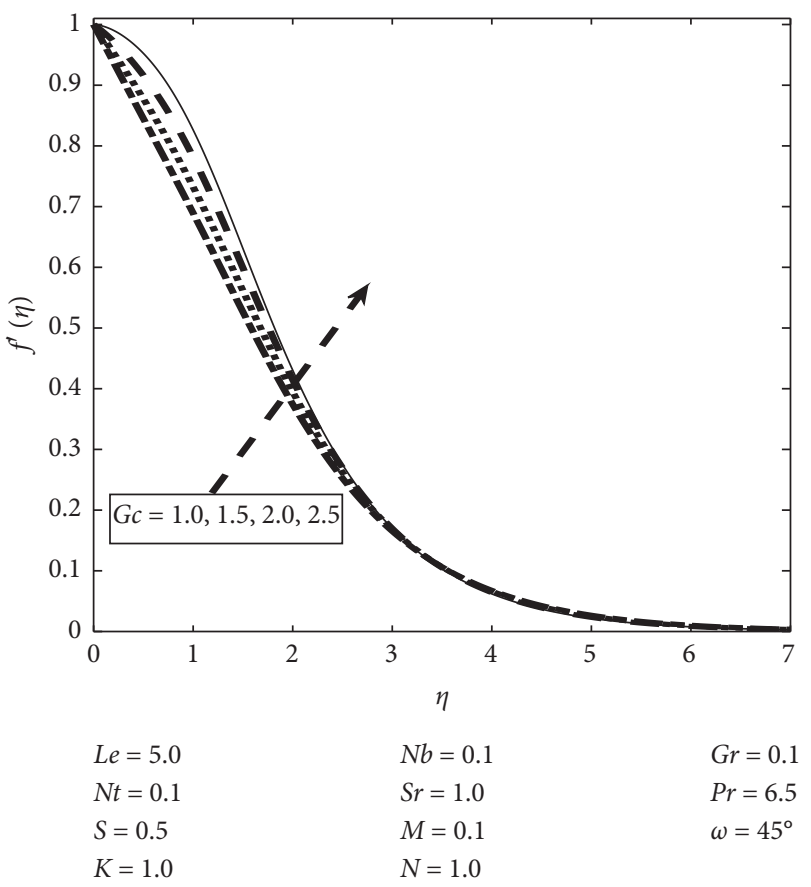

Figure 6: Velocity profile for several values of Gc.

motion effect as shown in Figure 12. Physically, the Brownian motion constraint improves the boundary layer heat which leads to rises in the fluid temperature. The variation between reference temperature and wall temperature is enhanced by growing the thermophoresis influence which corresponds the enhancement in temperature profile (Figure 13).

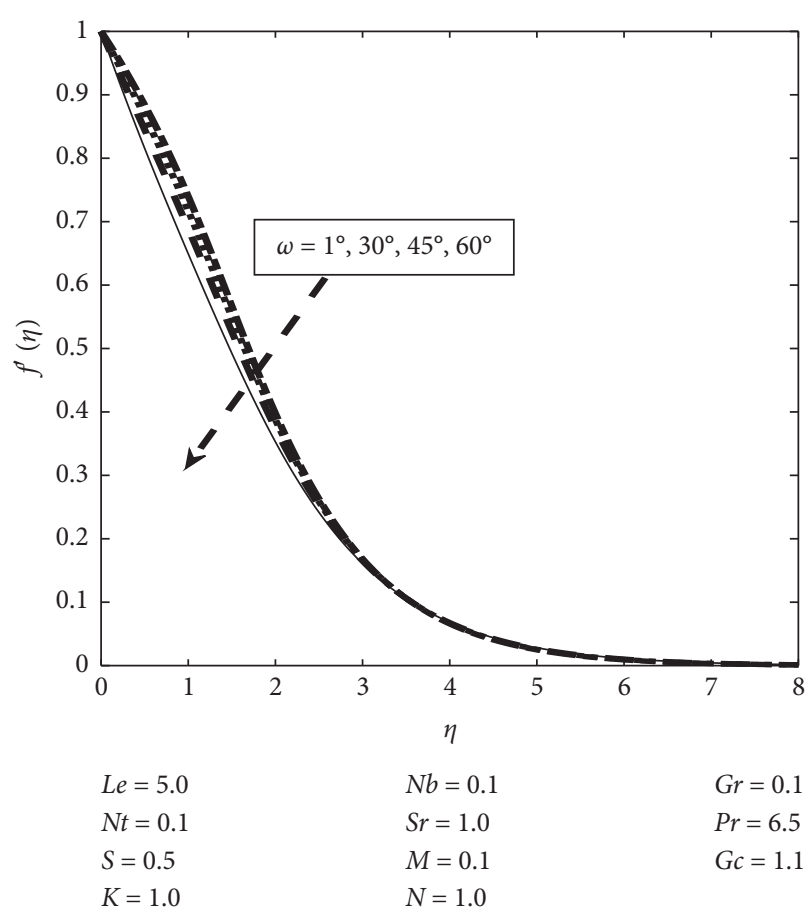

FIGURE 7: Velocity profile for several values of $\omega$.

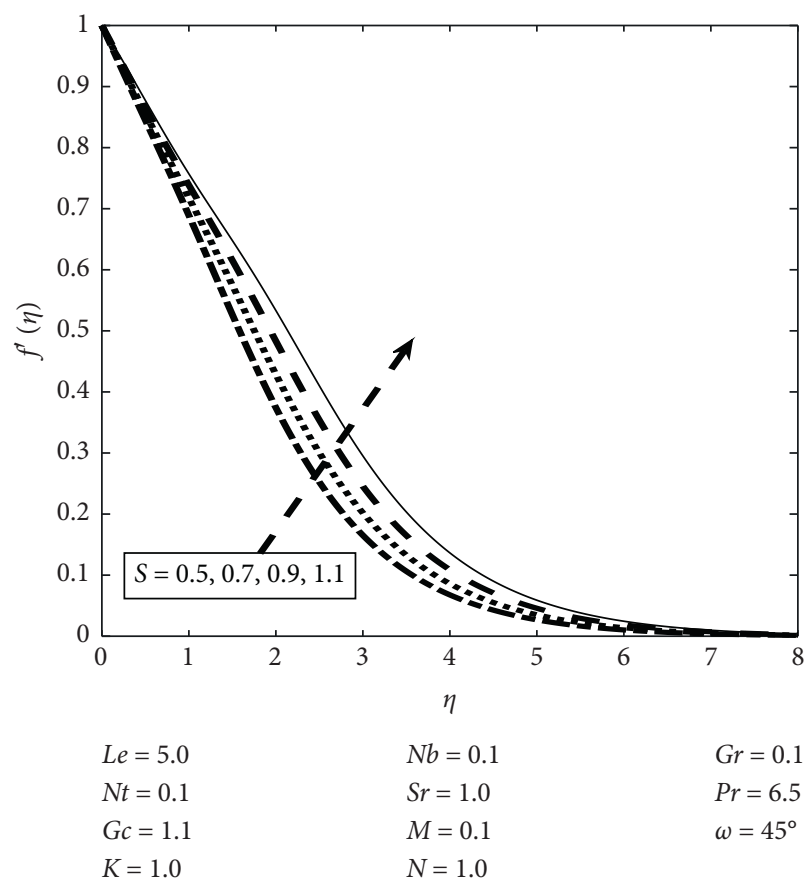

FIgURE 8: Velocity profile for several values of $S$.

3.2. Concentration. Figures 14 to 18 indicate concentration profiles against different incorporated parameters. The concentration profile improves on strengthening the magnetic impact as shown in Figure 14. An increment in Brownian motion parameter declines the concentration profile and the boundary layer thickness (Figure 15). Figure 16 presents more nanoparticles that pass away from the hot surface on enhancing 


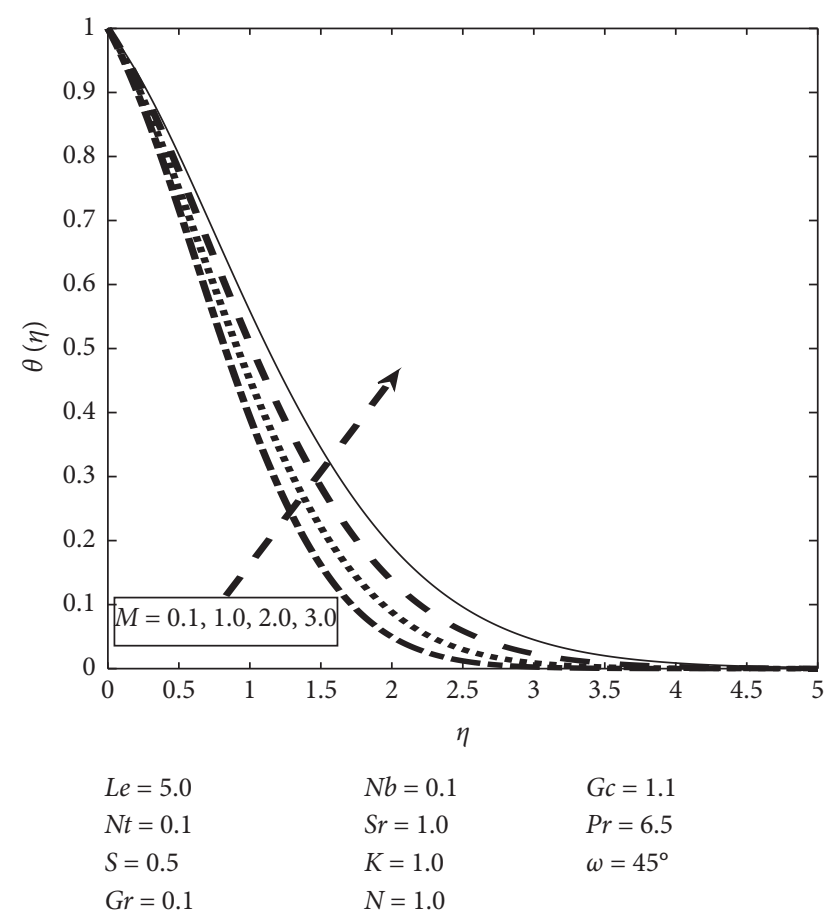

Figure 9: Temperature profile for several values of $M$.

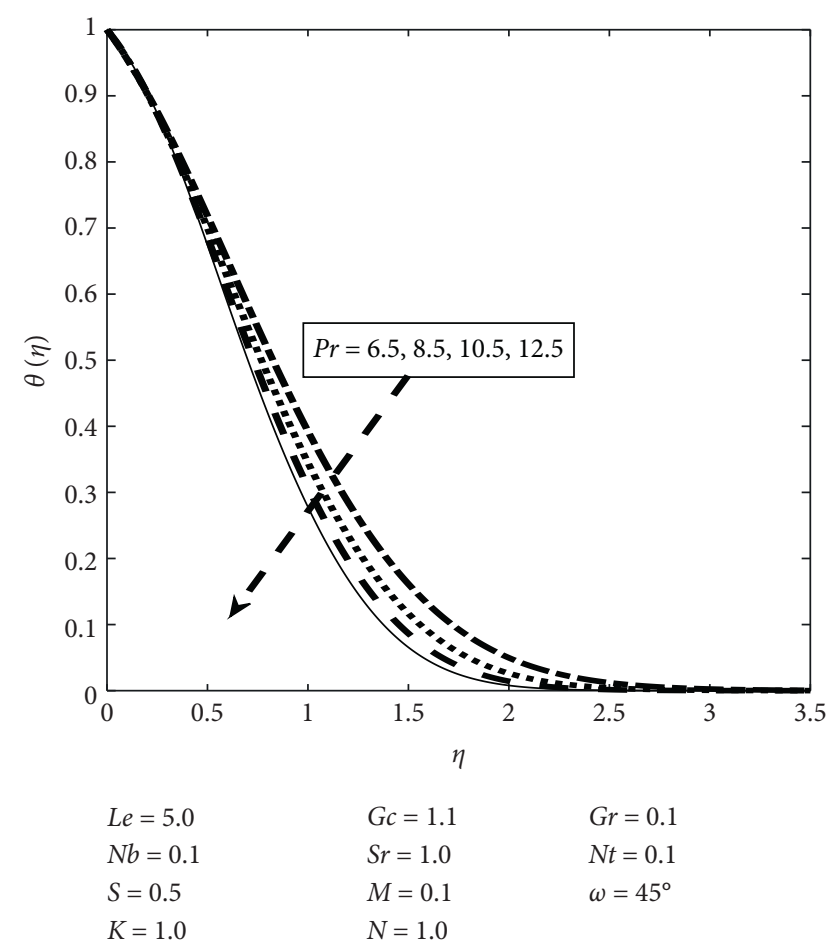

Figure 10: Temperature profile for several values of $\mathrm{Pr}$.

the thermophoretic effects which cause the improvement in the concentration contour. The boundary layer viscosity decreases against the Lewis number which relates to a drop in the concentration profile (Figure 17). The concentration profile corresponds to a direct variation with the Soret factor drawn in Figure 18.

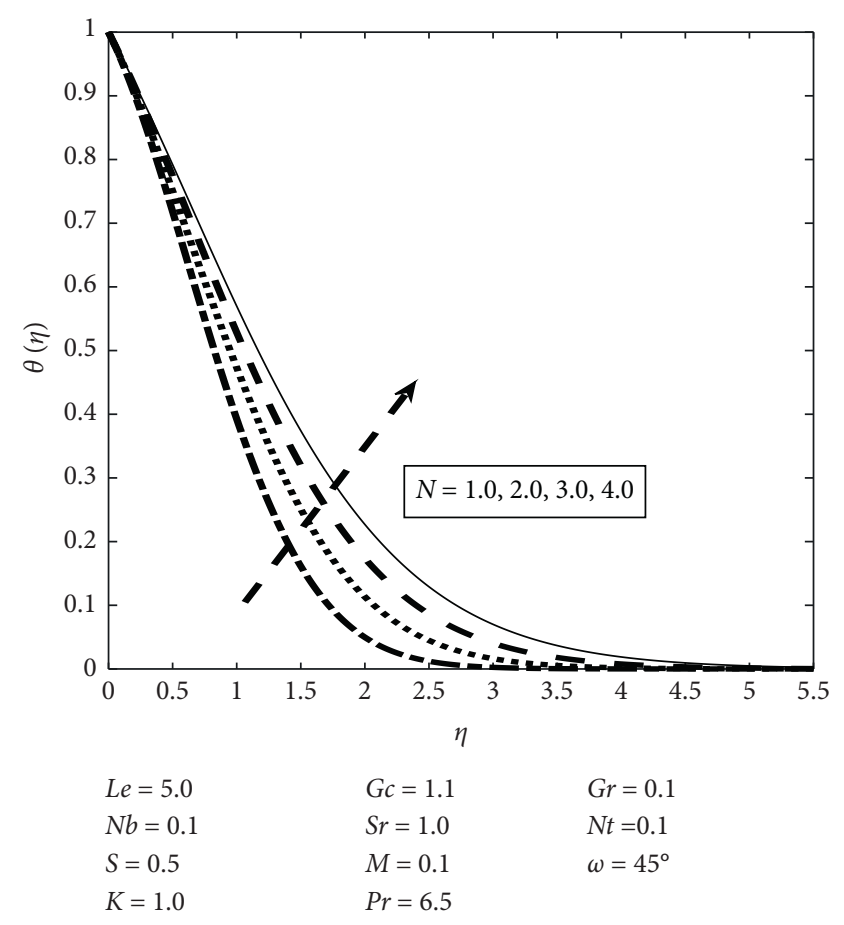

FIgURE 11: Temperature profile for several values of $N$.

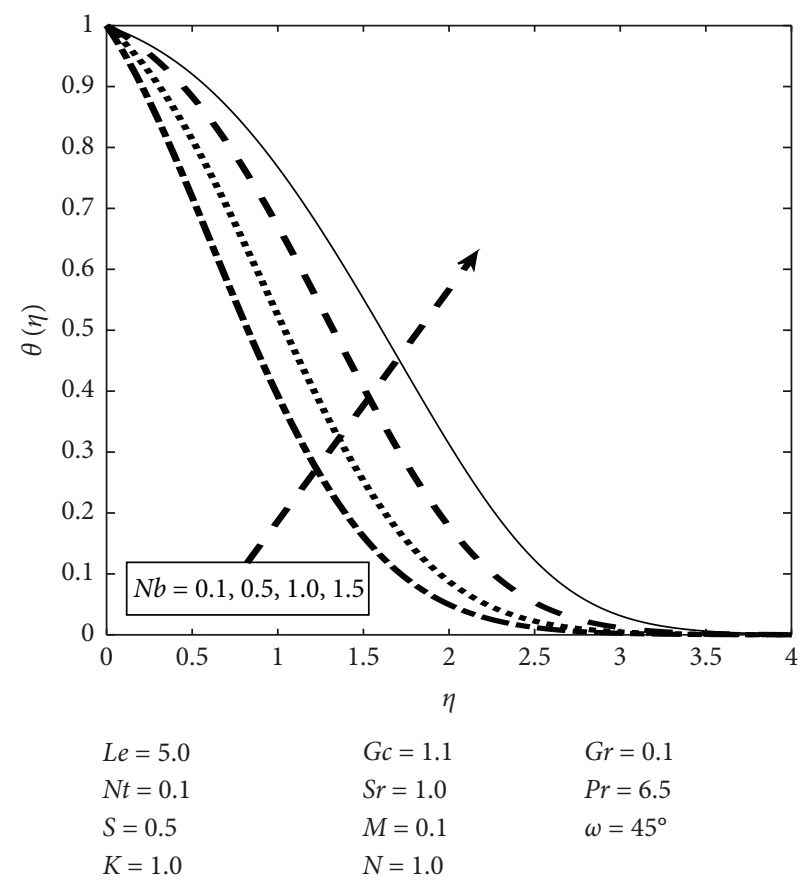

Figure 12: Temperature profile for several values of $\mathrm{Nb}$.

3.3. Heat and Mass Exchange. In order to check the behavior of dimensionless heat and mass exchange rates at the wall along with skin friction against the involved parameters, i.e., $\mathrm{Nb}$ and $\omega$, Figures 19 to 21 are drawn. Figure 19 reveals the impact of the Brownian motion factor against different inclination parameters. It is noted that $-\theta^{\prime}(0)$ inversely relates the Brownian motion factor and inclination factor. As 


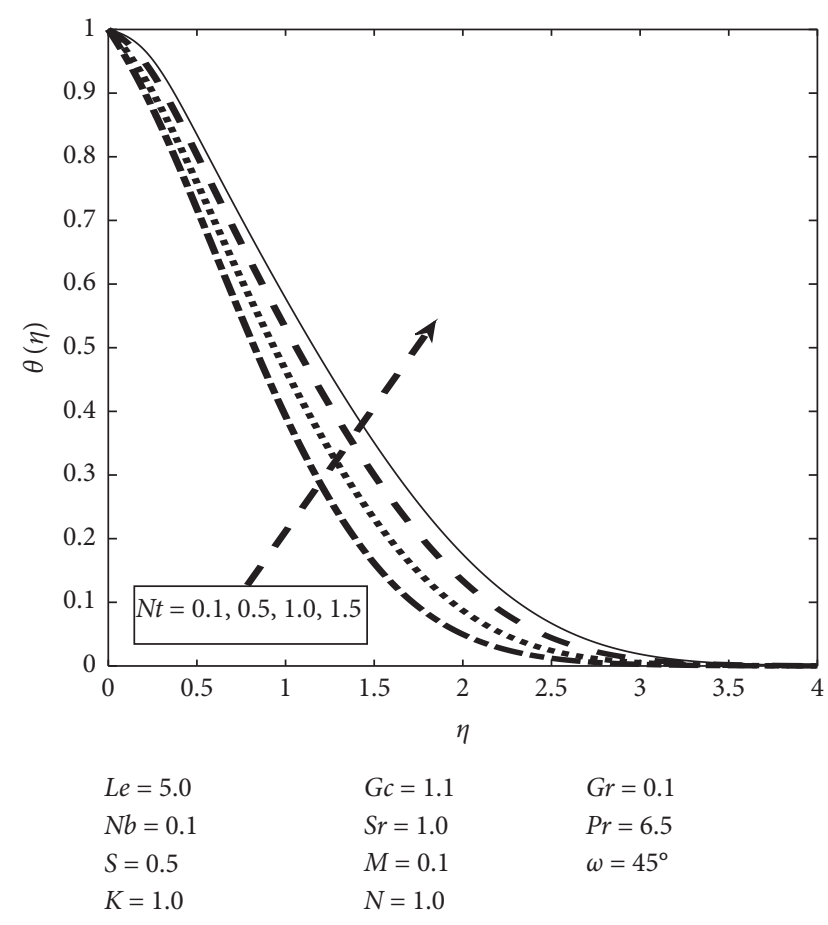

Figure 13: Temperature profile for several values of $N t$.

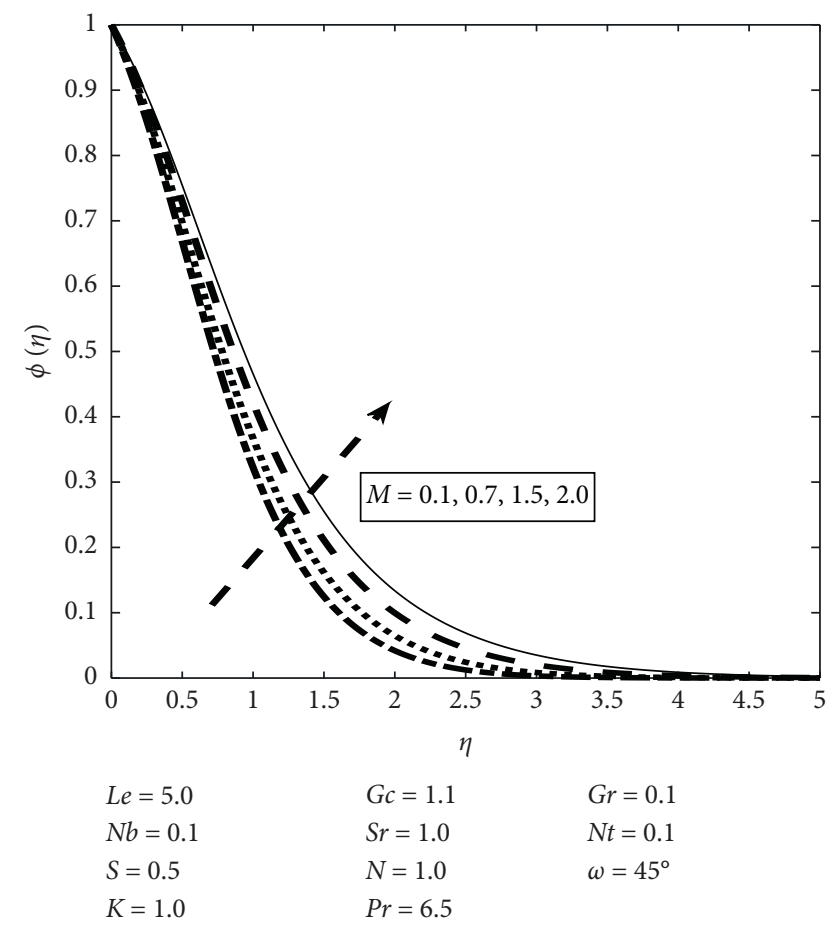

FIGURE 14: Concentration profile for several values of $M$.

we improve the inclination and the Brownian motion factor, the heat transfer rate decreases. The same impacts of the Brownian motion factor along with the inclination parameter on the mass exchange rate have been noticed in Figure 20. However, $C_{f x}(0)$ enhances for higher magnitudes of Brownian motion and inclination depicted in Figure 21.

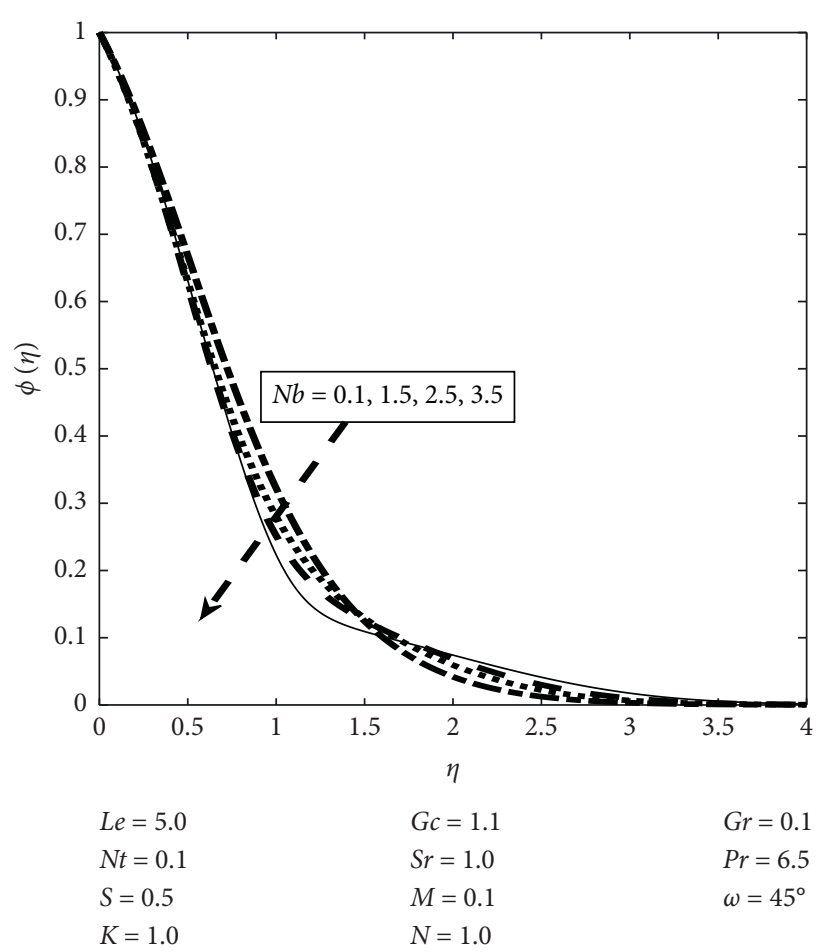

Figure 15: Concentration profile for several values of $\mathrm{Nb}$.

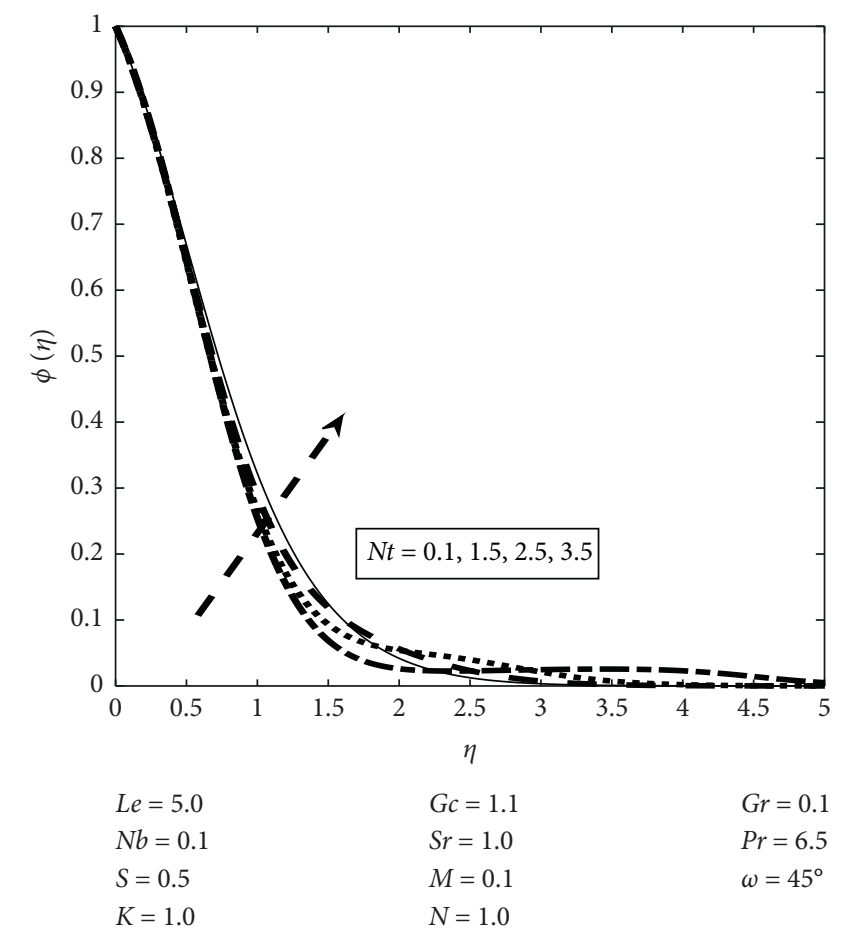

Figure 16: Concentration profile for several values of $\mathrm{Nt}$.

Figures 22 to 24 reveal the variations in $-\theta^{\prime}(0),-\phi^{\prime}(0)$, and $C_{f x}(0)$ versus $N t$ and $\omega$. On the contrary, heat exchange declines on the higher values of thermophoretic effect and inclination (Figure 22). Moreover, the mass exchange rate shows similar behavior like heat transfer rate against altered 


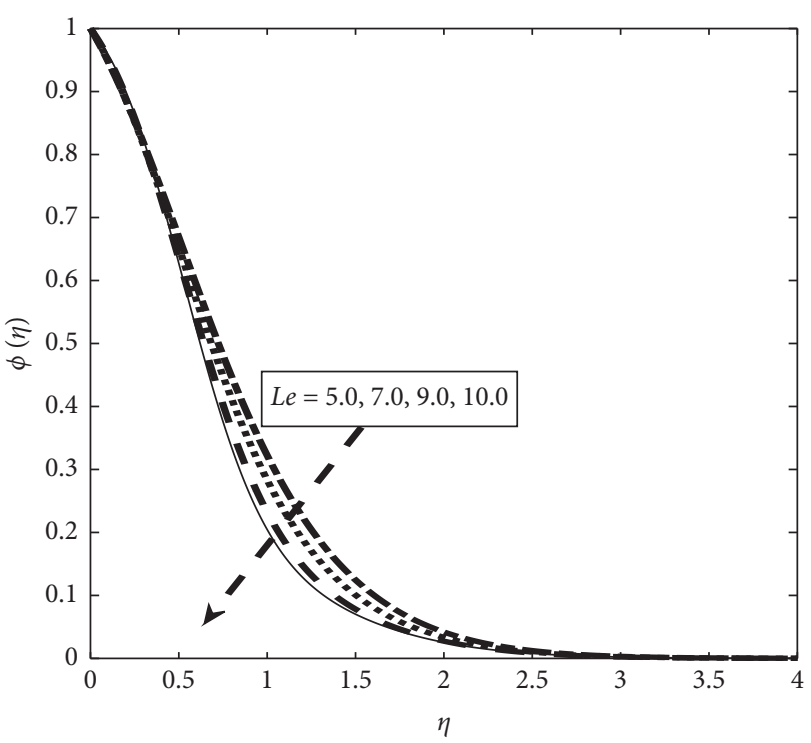

$$
\begin{array}{lll}
\operatorname{Pr}=6.5 & G c=1.1 & G r=0.1 \\
N b=0.1 & S r=1.0 & N t=0.1 \\
S=0.5 & M=0.1 & \omega=45^{\circ}
\end{array}
$$

Figure 17: Concentration profile for several values of $L e$.

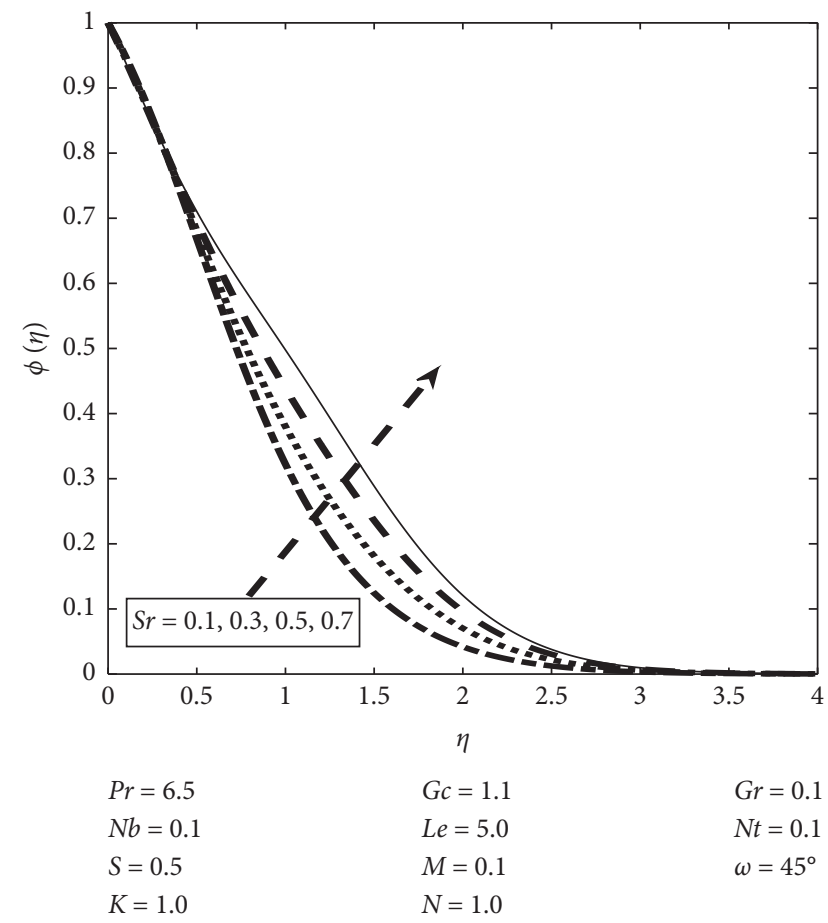

FIgURe 18: Concentration profile for several values of $S r$.

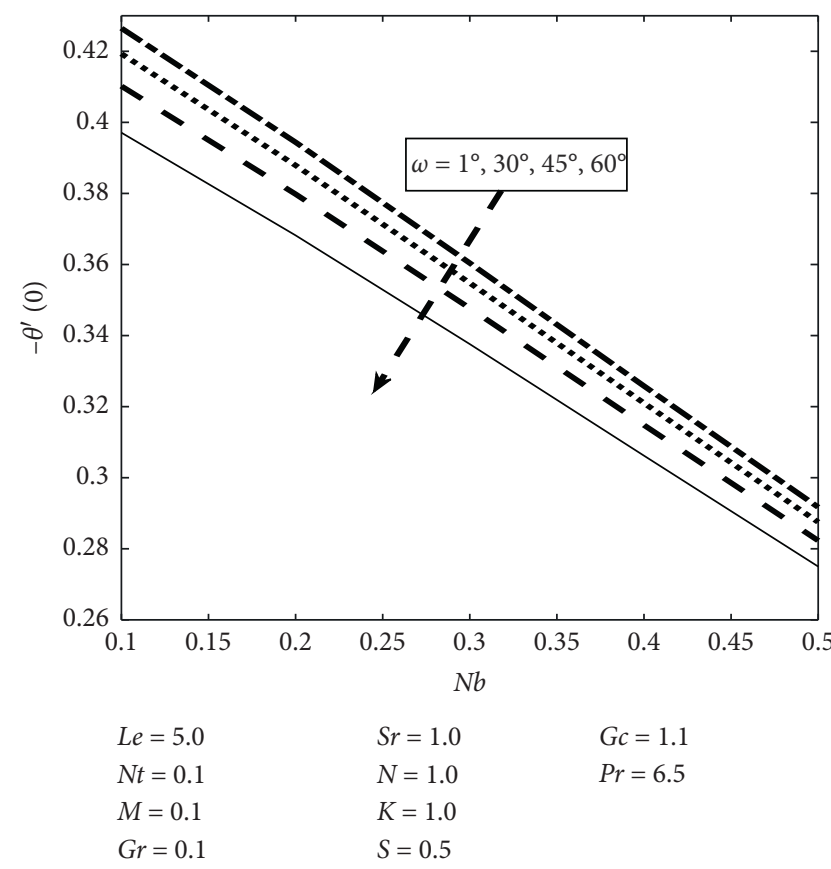

FIgURE 19: Variations of reduced Nusselt number with $\mathrm{Nb}$ for different values of $\omega$.

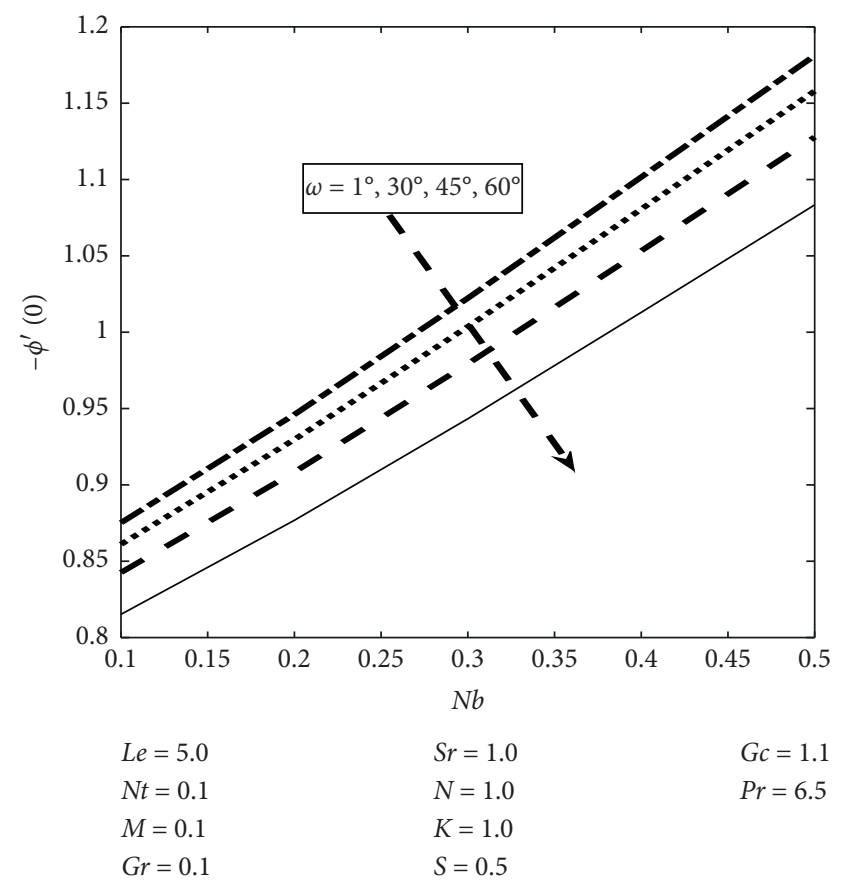

Figure 20: Variations of reduced Sherwood number with $\mathrm{Nb}$ for different values of $\omega$. 


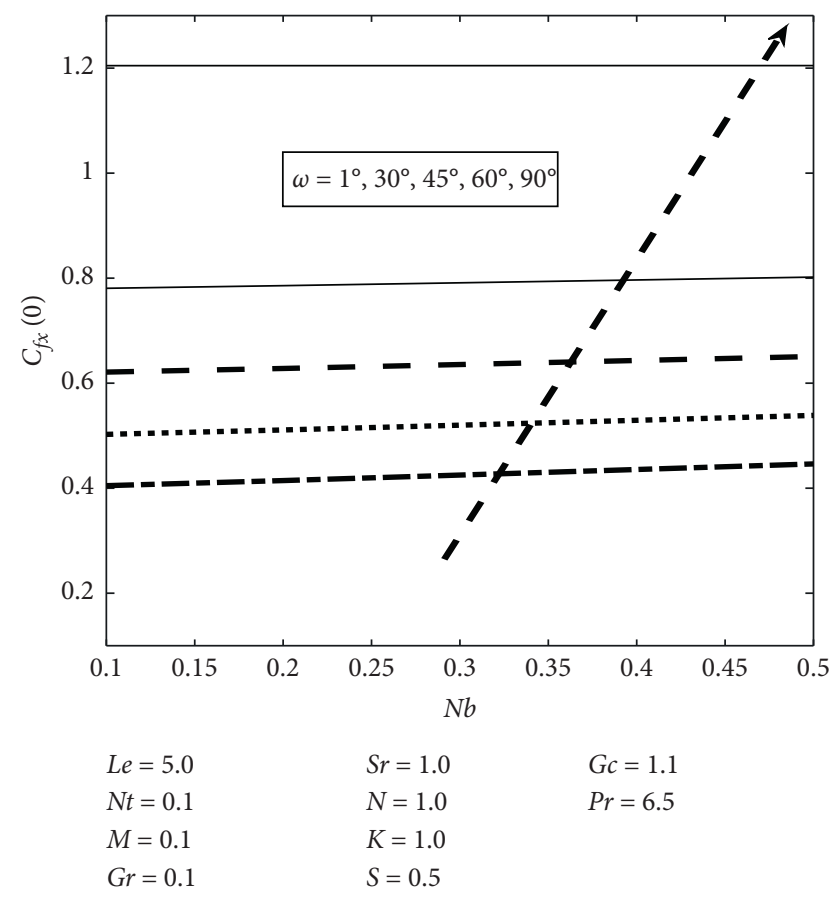

FIGURE 21: Variations skin friction with $N b$ for different values of $\omega$.

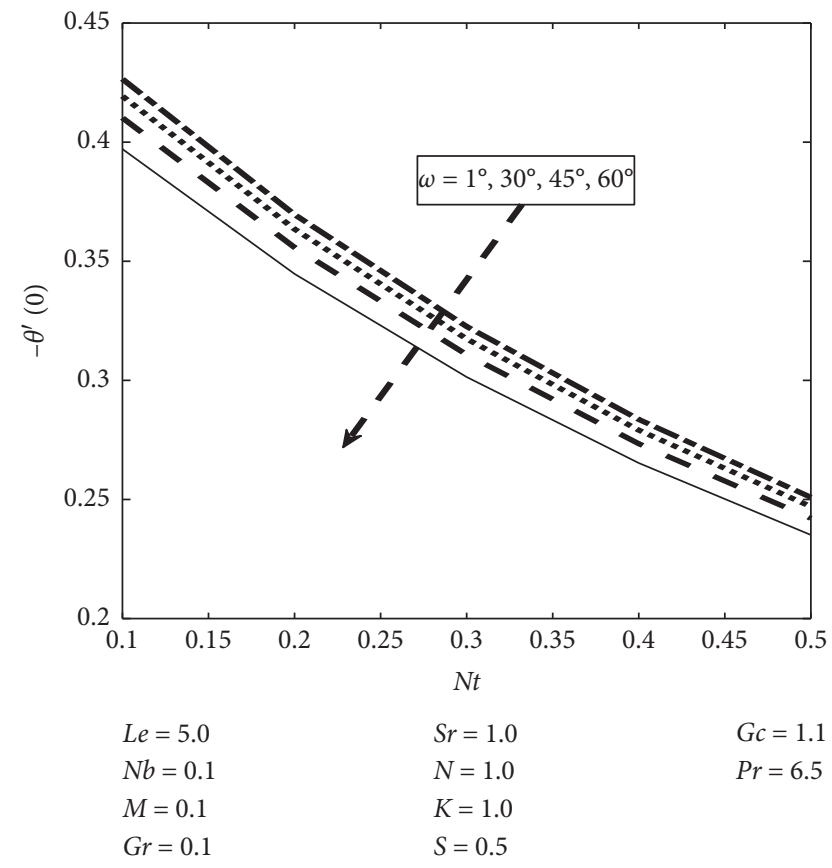

FIgURE 22: Variations of reduced Nusselt number with $N t$ for different values of $\omega$.

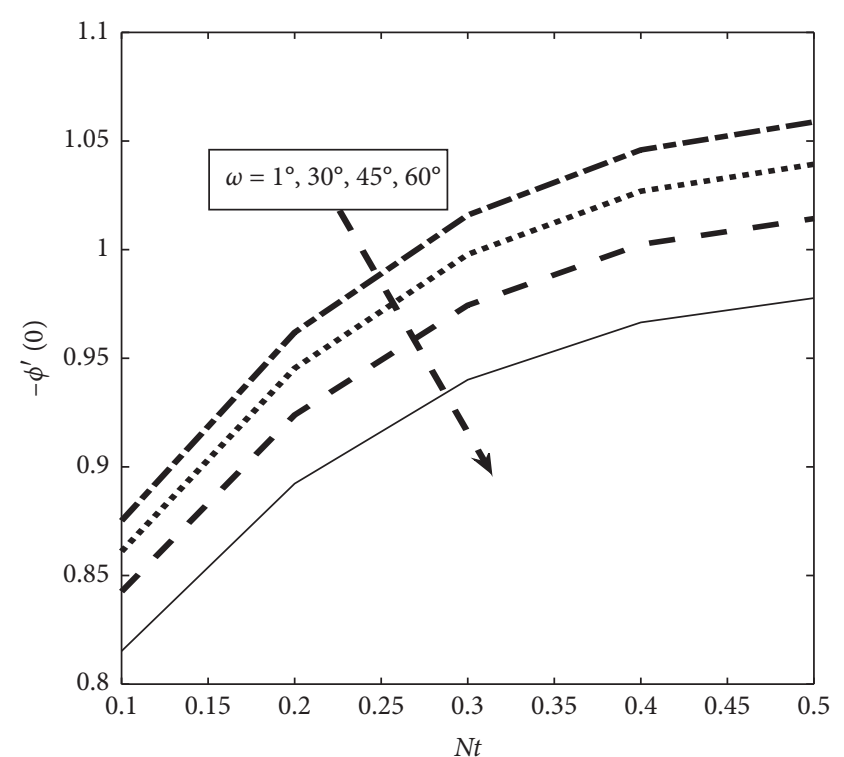

$\begin{array}{lll}L e=5.0 & S r=1.0 & G c=1.1 \\ N b=0.1 & N=1.0 & \operatorname{Pr}=6.5 \\ M=0.1 & K=1.0 & \\ G r=0.1 & S=0.5 & \end{array}$

Figure 23: Variations of reduced Sherwood number with $N t$ for different values of $\omega$.

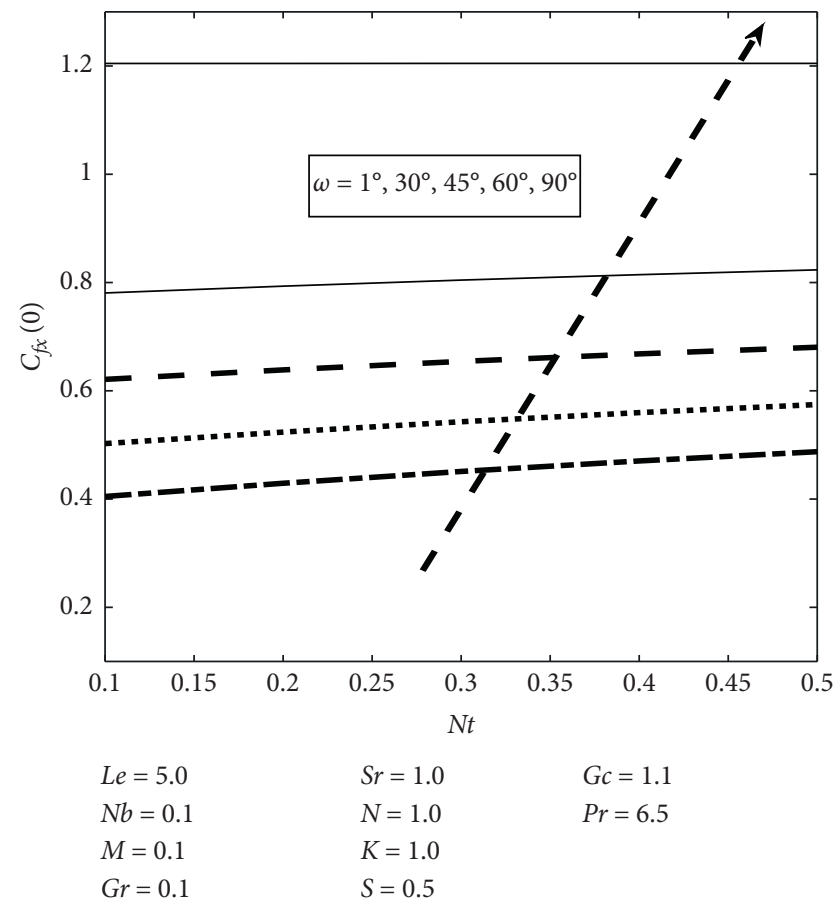

FIGURE 24: Variations skin friction with $N t$ for different values of $\omega$. 
values of inclination and thermophoresis impacts in Figure 23. In addition, skin friction is improved by enhancing the magnitudes of inclination and thermophoretic impacts (Figure 24).

\section{Conclusions}

The article presents numerical simulations of the micropolartype nanofluid flow over a slanted surface. Similarity results for velocity, temperature, and concentration are recovered through the Keller box technique. The statistical outcomes are compared with the already available literature. In research field, some notable points from this study are as follows:

(i) The irregular movement of particles boosts with the increment in Brownian motion; and as a result energy and species exchange rate diminish, whereas the skin friction is enhanced.

(ii) The temperature field is more influenced by increasing thermal radiation.

(iii) The concentration profile enhances with the growing magnitude of Soret impact.

(iv) The boundary layer viscosity decreases against Lewis number which relates to a drop in the concentration profile

(v) The conductive heat exchange is greater than the radiative heat exchange, which causes reduction in boundary layer thickness and buoyancy force.

\section{Nomenclaure}

C: $\quad$ Fluid concentration

Sr: Soret effect parameter

$N$ : Radiation parameter

$C_{f}$ : Skin friction coefficient

$S: \quad$ Suction/injection

$\mathrm{Re}_{x}$ : Reynolds number

$C_{\infty}$ : Nanoparticle volume fraction

Le: Lewis number

Sh: Sherwood number

$C_{w}$ : Surface volume fraction

$N b$ : Brownian motion parameter

T: $\quad$ Fluid temperature

$c_{p}$ : Specific heat at constant pressure

$N t$ : Thermophoretic parameter

$T_{w}$ : Wall temperature

$D_{B}$ : Brownian diffusion coefficient

$N u$ : Nusselt number

$T_{\infty}$ : Ambient temperature

$D_{T}$ : Thermophoretic diffusion coefficient

Pr: Prandtle number

$u_{w}$ : Wall velocity

$f$ : Similarity function for velocity

$g$ : Gravitational acceleration

$u_{\infty}$ : Ambient velocity

$\rho c_{p}$ : Volume heat capacity

$\mu$ : $\quad$ Kinematic viscosity

$v$ : Dynamic viscosity $\varphi: \quad$ Dimensionless solid volume fraction

$w$ : Condition at the wall

$\infty$ : Ambient condition

$G r$ : Local Grashof number

$\beta_{t}$ : Thermal expansion coefficient

$\beta_{c}$ : Concentration expansion coefficient

$\sigma$ : $\quad$ Electric conductivity

$\gamma^{*}$ : Spin gradient viscosity

$k_{1}^{*}$ : Vertex viscosity

$j^{*}$ : $\quad$ Microinertia per unit mass

$\gamma: \quad$ Inclination parameter

1: Differentiation with respect to $\eta$

$u$ : $\quad$ Velocity in $x$ direction

$v$ : Velocity in $y$ direction

$x$ : Cartesian coordinate

$\rho$ : $\quad$ Fluid density

$a$ : Stretching rate

$k$ : Thermal conductivity

$K$ : $\quad$ Material parameter

Gc: Modified local Grashof number

$B_{0}$ : Uniform magnetic field strength

$\theta$ : Dimensionless temperature

$\eta$ : $\quad$ Similarity independent variable

$\alpha$ : Thermal diffusivity

$N^{*}$ : Nondimensional angular velocity.

\section{Data Availability}

The data used to support the findings of this study are available from the corresponding author upon request.

\section{Conflicts of Interest}

The authors declare that they have no conflicts of interest.

\section{Authors' Contributions}

K. R. formulated the problem. H. A. elucidated the numerical results. T. A. N discussed the physical parameters. M. I. A. prepared the manuscript final draft. M. M. checked the numerical results, and I.K verified the results and proof read it.

\section{Acknowledgments}

This work was supported by Taif University researches supporting project number (TURSP-2020/031), Taif University, Saudi Arabia.

\section{References}

[1] K. Rafique, M. Imran Anwar, and M. Misiran, "Keller-box study on casson nano fluid flow over a slanted permeable surface with chemical reaction," Asian Research Journal of Mathematics, vol. 14, no. 4, pp. 1-17, 2019.

[2] A. Saeed, Z. Shah, S. Islam et al., "Three-dimensional casson nanofluid thin film flow over an inclined rotating disk with the impact of heat generation/consumption and thermal radiation," Coatings, vol. 9, no. 4, p. 248, 2019.

[3] M. S. Rani and A. Govindarajan, "Radiative fluid flow of a nanofluid over an inclined plate with non-uniform surface 
temperature," Journal of Physics: Conference Series, vol. 1000, no. 1, p. 012173, 2018.

[4] A. Khan, D. Khan, I. Khan, F. Ali, F. ul Karim, and M. Imran, "MHD flow of sodium alginate-based casson type nanofluid passing through a porous medium with newtonian heating," Scientific Reports, vol. 8, no. 1, p. 8645, 2018.

[5] P. Suriyakumar and S. P. A. Devi, "Effects of suction and internal heat generation on hydromagnetic mixed convective nanofluid flow over an inclined," Stretching Plate, vol. 2, no. 3, pp. 51-58, 2015.

[6] M. Khan, A. Shahid, M. Y. Malik, and T. Salahuddin, "Thermal and concentration diffusion in Jeffery nanofluid flow over an inclined stretching sheet: a generalized Fourier's and Fick's perspective," Journal of Molecular Liquids, vol. 251, pp. 7-14, 2018.

[7] T. Thumma, O. Anwar Bég, and A. Kadir, "Numerical study of heat source/sink effects on dissipative magnetic nanofluid flow from a non-linear inclined stretching/shrinking sheet," Journal of Molecular Liquids, vol. 232, pp. 159-173, 2017.

[8] A. Rashad, "Unsteady nanofluid flow over an inclined stretching surface with convective boundary condition and anisotropic slip impact," International Journal of Heat and Technology, vol. 35, no. 1, pp. 82-90, 2017.

[9] M. Hatami, D. Jing, and M. A. Yousif, "Three-dimensional analysis of condensation nanofluid film on an inclined rotating disk by efficient analytical methods," Arab Journal of Basic and Applied Sciences, vol. 25, no. 1, pp. 28-37, 2018.

[10] H. Alotaibi, S. Althubiti, M. R. Eid, and K. L. Mahny, "Numerical treatment of mhd flow of casson nanofluid via convectively heated non-linear extending surface with viscous dissipation and suction/injection effects," Computers, Materials \& Continua, vol. 66, no. 1, pp. 229-245, 2020.

[11] D. Pal and N. Roy, "Role of brownian motion and nonlinear thermal radiation on heat transfer of a casson nanofluid over stretching sheet with slip velocity and non-uniform heat source/sink," Journal of Nanofluids, vol. 8, no. 3, pp. 556-568, 2019.

[12] S. S. Ghadikolaei, K. Hosseinzadeh, D. D. Ganji, and B. Jafari, "Nonlinear thermal radiation effect on magneto Casson nanofluid flow with Joule heating effect over an inclined porous stretching sheet," Case Studies in Thermal Engineering, vol. 12, pp. 176-187, 2018.

[13] N. Saidulu, T. Gangaiah, and A. V. Lakshmi, "Radiation effect ON MHD flow OF a tangent hyperbolic nanofluid over an inclined exponentially stretching sheet," International Journal of Fluid Mechanics Research, vol. 46, no. 3, 2019.

[14] C. Sulochana, S. P. Samrat, and N. Sandeep, "Magnetohydrodynamic radiative nanofluid flow over a rotating surface with soret effect," Multidiscipline Modeling in Materials and Structures, vol. 14, no. 1, pp. 168-188, 2018.

[15] M. R. Eid and O. D. Makinde, "Solar radiation effect on a magneto nanofluid flow in a porous medium with chemically reactive species," International Journal of Chemical Reactor Engineering, vol. 16, no. 9, 2018.

[16] T. Chakraborty, K. Das, and P. K. Kundu, "Ag-water nanofluid flow over an inclined porous plate embedded in a nonDarcy porous medium due to solar radiation," Journal of Mechanical Science and Technology, vol. 31, no. 5, pp. 24432449, 2017.

[17] M. A. Mjankwi, V. G. Masanja, E. W. Mureithi, and M. N. O. James, "Unsteady MHD flow of nanofluid with variable properties over a stretching sheet in the presence of thermal radiation and chemical reaction," International
Journal of Mathematics and Mathematical Sciences, vol. 2019, Article ID 7392459, 14 pages, 2019.

[18] K. Rafique, M. I. Anwar, M. Misiran et al., "Brownian motion and thermophoretic diffusion effects on micropolar type nanofluid flow with Soret and Dufour impacts over an inclined sheet: Keller-box simulations," Energies, vol. 12, no. 21, p. 4191, 2019.

[19] R. Ahmad, A. Farooqi, J. Zhang, and N. Ali, "Steady flow of a power law fluid through a tapered non-symmetric stenotic tube," Applied Mathematics and Nonlinear Sciences, vol. 4, no. 1, pp. 249-260, 2019.

[20] N. A. Sheikh, F. Ali, M. Saqib et al., "Comparison and analysis of the Atangana-Baleanu and Caputo-Fabrizio fractional derivatives for generalized Casson fluid model with heat generation and chemical reaction," Results in Physics, vol. 7, pp. 789-800, 2017.

[21] N. A. Sheikh, F. Ali, I. Khan, M. Saqib, and A. Khan, "MHD flow of micropolar fluid over an oscillating vertical plate embedded in porous media with constant temperature and concentration," Mathematical Problems in Engineering, vol. 2017, Article ID 9402964, 20 pages, 2017.

[22] N. A. Sheikh, D. L. Chuan Ching, I. Khan, A. Ahmad, and S. Ammad, "Concrete based jeffrey nanofluid containing zinc oxide nanostructures: application in cement industry," Symmetry, vol. 12, no. 6, p. 1037, 2020.

[23] F. Ali, N. A. Sheikh, I. Khan, and M. Saqib, "Magnetic field effect on blood flow of Casson fluid in axisymmetric cylindrical tube: a fractional model," Journal of Magnetism and Magnetic Materials, vol. 423, pp. 327-336, 2017.

[24] N. A. Sheikh, D. L. Chuan Ching, and I. Khan, "A comprehensive review on theoretical aspects of nanofluids: exact solutions and analysis," Symmetry, vol. 12, no. 5, p. 725, 2020.

[25] A. C. Eringen, "Simple microfluids," International Journal of Engineering Science, vol. 2, no. 2, pp. 205-217, 1964.

[26] M. Uddin, "Convective flow of micropolar fluids along an inclined flat plate with variable electric conductivity and uniform surface heat flux," Daffodil International University Journal of Science and Technology, vol. 6, no. 1, 2011.

[27] M. D. Shamshuddin and T. Thumma, "Numerical study of a dissipative micropolar fluid flow past an inclined porous plate with heat source/sink," Propulsion and Power Research, vol. 8, no. 1, pp. 56-68, 2019.

[28] E. Nandhini and M. Ramya, "MHD free convection flow in a micropolar fluid past an inclined stretching sheet with considering viscous dissipation and radiation," International Journal of Scientific Research in Science, Engineering and Technology, vol. 4, no. 1, pp. 1478-1485, 2018.

[29] A. R. M. Kasim, N. F. Mohammad, and S. Shafie, "Unsteady MHD mixed convection flow of a micropolar fluid along an inclined stretching plate," Heat Transfer-Asian Research, vol. 42, no. 2, pp. 89-99, 2013.

[30] M. M. Rahman, A. Aziz, and M. A. Al-lawatia, "Heat transfer in micropolar fluid along an inclined permeable plate with variable fluid properties," International Journal of Thermal Sciences, vol. 49, no. 6, pp. 993-1002, 2010.

[31] D. Srinivasacharya and K. Hima Bindu, "Entropy generation in a micropolar fluid flow through an inclined channel," Alexandria Engineering Journal, vol. 55, no. 2, pp. 973-982, 2016.

[32] D. Srinivasacharya, C. RamReddy, and P. Naveen, "Double dispersion effect on nonlinear convective flow over an inclined plate in a micropolar fluid saturated non-Darcy porous medium," Engineering Science and Technology, an International Journal, vol. 21, no. 5, pp. 984-995, 2018. 
[33] A. Hazbavi and S. Sharhani, "Micropolar fluid flow between two inclined parallel plates," in Proceedings of the ASME 2017 International Mechanical Engineering Congress and Exposition, November 2017.

[34] T. Hayat, M. I. Khan, M. Waqas, and A. Alsaedi, "Newtonian heating effect in nanofluid flow by a permeable cylinder," Results in Physics, vol. 7, pp. 256-262, 2017.

[35] W. A. Khan and I. Pop, "Boundary-layer flow of a nanofluid past a stretching sheet," International Journal of Heat and Mass Transfer, vol. 53, no. 11-12, pp. 2477-2483, 2010.

[36] M. I. Anwar, S. Shafie, T. Hayat, S. A. Shehzad, and M. Z. Salleh, "Numerical study for MHD stagnation-point flow of a micropolar nanofluid towards a stretching sheet," Journal of the Brazilian Society of Mechanical Sciences and Engineering, vol. 39, no. 1, pp. 89-100, 2017.

[37] M. I. Anwar, K. Rafique, M. Misiran, and S. A. Shehzad, "Numerical study of hydrodynamic flow of a Casson nanomaterial past an inclined sheet under porous medium," Heat Transfer-Asian Research, vol. 49, no. 1, pp. 307-334, 2020.

[38] A. A. Afify, "Similarity solution in MHD: effects of thermal diffusion and diffusion thermo on free convective heat and mass transfer over a stretching surface considering suction or injection," Communications in Nonlinear Science and Numerical Simulation, vol. 14, no. 5, pp. 2202-2214, 2009.

[39] K. Rafique, M. I. Anwar, M. Misiran et al., "Keller-box simulation for the buongiorno mathematical model of micropolar nanofluid flow over a nonlinear inclined surface," Processes, vol. 7, no. 12, p. 926, 2019.

[40] O. D. Makinde and P. O. Olanrewaju, "Buoyancy effects on thermal boundary layer over a vertical plate with a convective surface boundary condition," Journal of Fluids Engineering, vol. 132, no. 4, 2010.

[41] M. R. Ilias, N. A. Rawi, and S. Shafie, "MHD free convection flow and heat transfer of ferrofluids over a vertical flat plate with aligned and transverse magnetic field," Indian Journal of Science and Technology, vol. 9, no. 36, pp. 1-7, 2016.

[42] K. Rafique, M. Imran Anwar, M. Misiran et al., "Keller-box analysis of buongiorno model with brownian and thermophoretic diffusion for casson nanofluid over an inclined surface," Symmetry, vol. 11, no. 11, p. 1370, 2019. 